\title{
Modeling Acute Health Effects of Astronauts from Exposure to Large Solar Particle Events
}

Shaowen $\mathrm{Hu}^{1}$, Myung-Hee Y. Kim ${ }^{1}$, and Francis A. Cucinotta ${ }^{2}$

${ }^{1}$ Universities Space Research Association, Division of Space Life Sciences, Houston, Texas 77058, USA, and

${ }^{2}$ NASA, Lyndon B. Johnson Space Center, 2101 NASA Parkway, Houston Texas 77058, USA

In space exploration outside the Earth's geomagnetic field, radiation exposure from solar particle events (SPE) presents a health concern for astronauts, that could impair their performance and result in possible failure of the mission. Acute risks are of special concern during extra-vehicular activities because of the rapid onset of SPE. However, most SPEs will not lead to acute risks but can lead to mission disruption if accurate projection methods are not available. Acute Radiation Sickness (ARS) is a group of clinical syndromes developing acutely (within several seconds to 3 days) after high dose whole-body or significant partial-body ionizing radiation exposures. The manifestation of these syndromes reflects the disturbance of physiological processes of various cellular groups damaged by radiation. Hematopoietic cells, skin, epithelium, intestine, and vascular endothelium are among the most sensitive tissues of human body to ionizing radiation. Most ARS symptoms are directly related to these tissues and other systems (nervous, endocrine, and cardiovascular, etc.) with coupled regulations. Here we report the progress in bio-mathematical models to describe the dose and time-dependent early human responses to ionizing radiation. The responses include lymphocyte depression, granulocyte modulation, fatigue and weakness syndrome, and upper gastrointestinal distress. The modest dose and dose-rates of SPEs are predicted to lead to large sparing of ARS, however detailed experimental data on a range of proton dose-rates for organ doses from 0.5 to 2 Gy is needed to validate the models. We also report on the ARRBOD code that integrates the BRYNTRN and SUMDOSE codes, which are used to estimate the SPE organ doses for astronauts under various space travel scenarios, with our models of ARS. The more recent effort is to provide easy web access to space radiation risk assessment using the ARRBOD code. 


\section{Modeling Acute Health Effects of Astronauts from Exposure to Large Solar Particle Events}

Shaowen $\mathrm{Hu}^{1}$, Myung-Hee Y. Kim¹, and Francis A. Cucinotta ${ }^{2}$

${ }^{1}$ Universities Space Research Association, Division of Space Life Sciences, Houston, Texas 77058, USA, ${ }^{2}$ NASA, Lyndon B. Johnson Space Center, Houston, Texas 77058, USA 


\section{NASA Space Radiation Program Goal:}

\section{To live and work safely in space with acceptable risks from radiation}

Risk is not measured-It is predicted by a model 


\section{Acute irradiation syndrome}

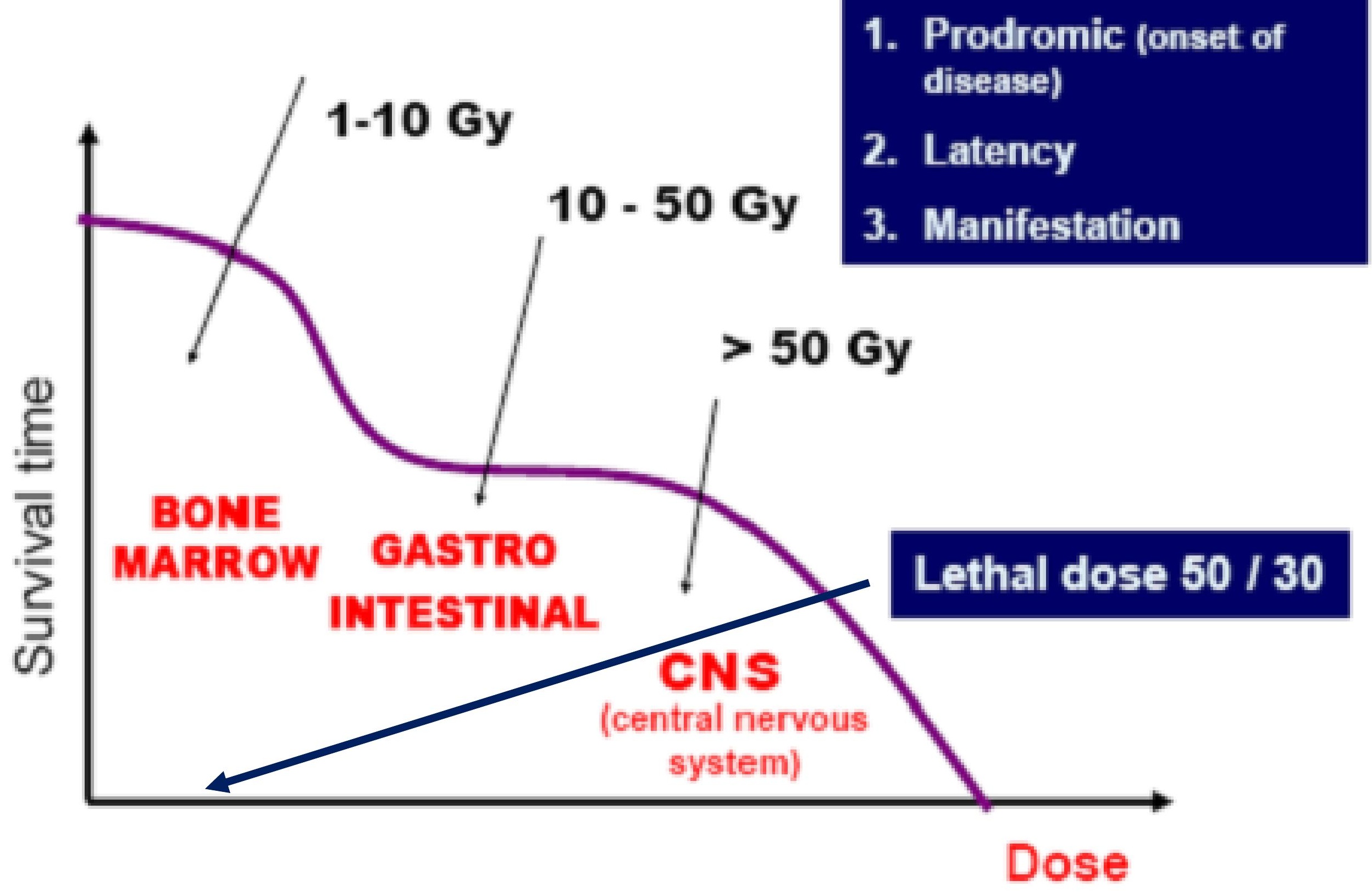

\section{Steps:}

1. Prodromic (onset of disease) 


\section{Correlation of time of death with irradiation anemia of different species}

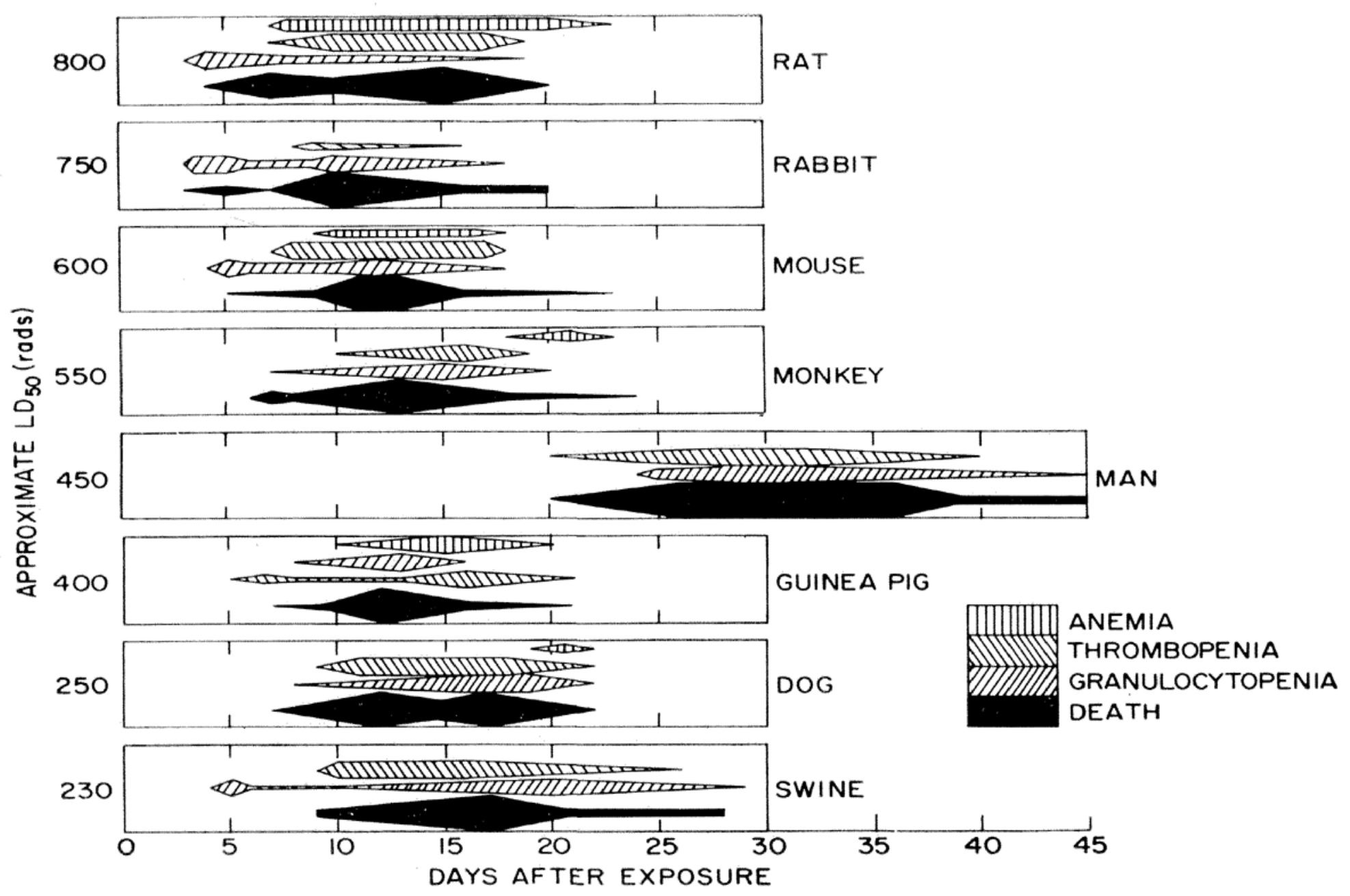




\section{Hematopoiesis in human}

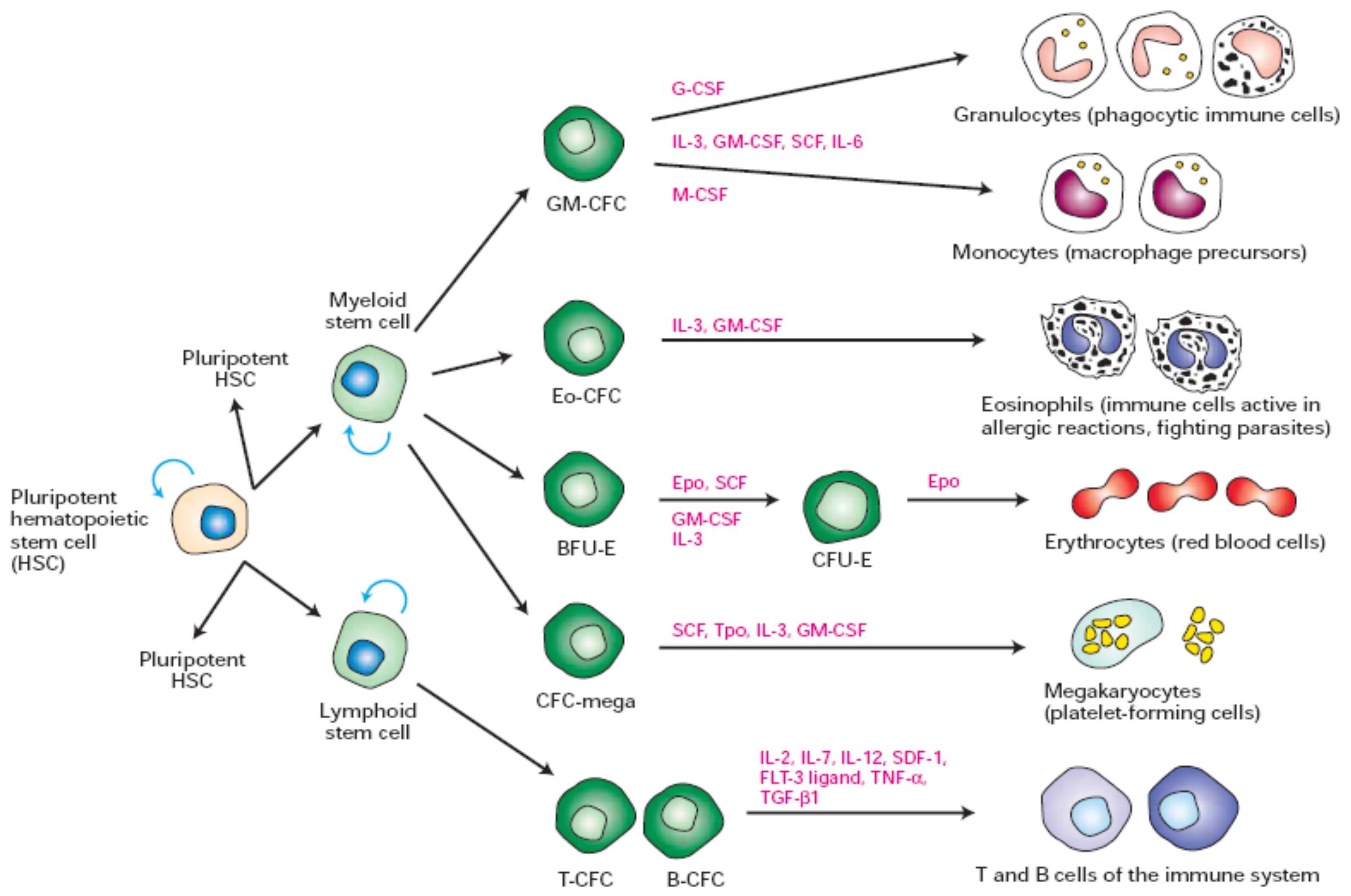




\section{Basis of mathematical modeling: regulation mechanism}

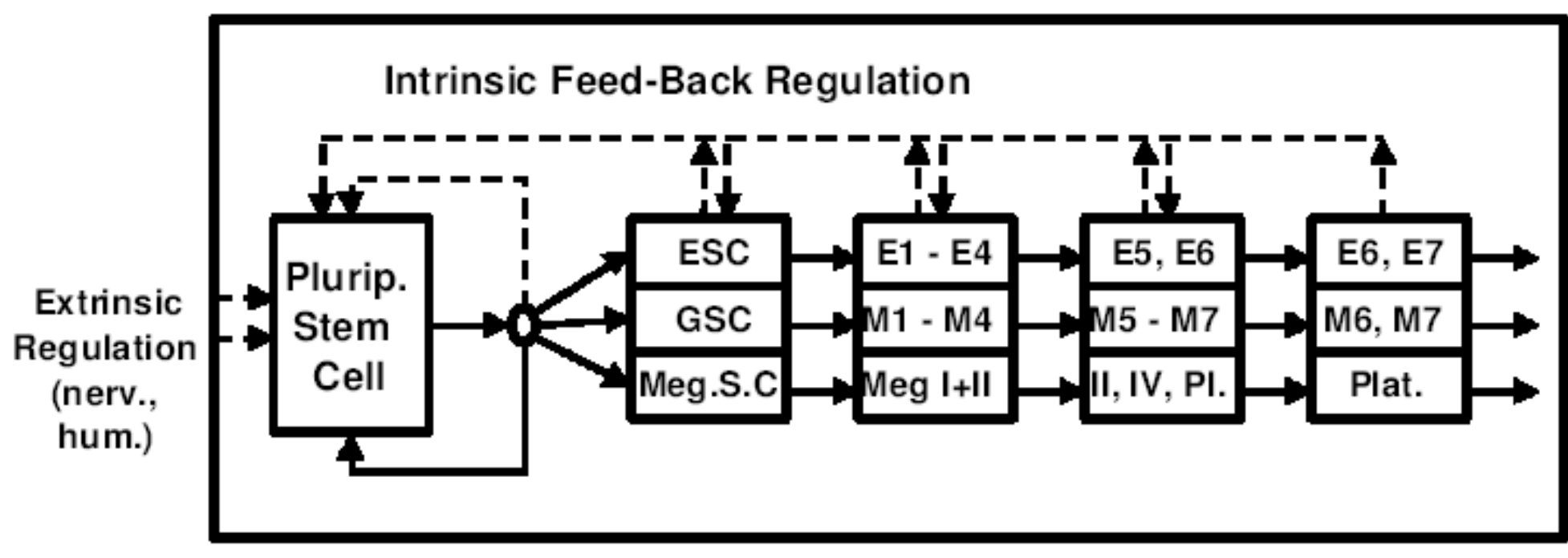

\begin{tabular}{|c|c|c|c|c|}
\hline $\begin{array}{l}\text { Uncommitted } \\
\text { stem cell } \\
\text { pool }\end{array}$ & $\begin{array}{c}\text { Committed } \\
\text { Stem Cell } \\
\text { Pool }\end{array}$ & $\begin{array}{c}\text { Proliferative } \\
\text { and } \\
\text { Maturing } \\
\text { Pool }\end{array}$ & $\begin{array}{c}\text { Maturing- } \\
\text { only and } \\
\text { storage } \\
\text { pool }\end{array}$ & $\begin{array}{c}\text { Functional } \\
\text { pool }\end{array}$ \\
\hline
\end{tabular}

Figure 2. Schematic representation of the hemopoietic cell renewal systems $(\mathrm{E}=$ Erythropoiesis; $\mathrm{G}=$ Granulopoiesis; Meg $=$ Megakaryocytopoiess) all fed by the pluripotent hematopoietic stem cell pool. 


\section{Smirnova's model}

- Three compartments:

- $X_{1}$ : bone marrow precursor cells

$-X_{2}$ : nondividing maturing bone marrow cells

- $X_{3}$ : mature blood cells

- Feed-back regulator $I$ : dependent on the concentration of $X_{1}, X_{2}$, and $X_{3}$ cells
Model equations:

$$
\frac{d x_{1}}{d t}=B x_{1}-C x_{1},
$$

$\frac{d x_{2}}{d t}=C x_{1}-F x_{2}$,

$\frac{d x_{3}}{d t}=F x_{2}-E x_{3}$,

$\frac{d I}{d t}=G\left(x_{1}+\theta_{2} x_{2}+\theta_{3} x_{3}\right)-H I$. 


\section{Granulocytopoiesis}

$$
\begin{aligned}
& \frac{d x_{1}}{d t}=\frac{\alpha x_{1}}{1+\beta\left(x_{1}+\theta_{2} x_{2}+\theta_{4} x_{4}+\theta_{5} x_{5}\right)}-\gamma x_{1}, \\
& \frac{d x_{2}}{d t}=\gamma x_{1}-\delta \frac{1+M x_{4}^{2}}{1+L x_{4}^{2}} x_{2}, \\
& \frac{d x_{4}}{d t}=\delta \frac{1+M x_{4}^{2}}{1+L x_{4}^{2} x_{2}-\kappa x_{4},} \begin{array}{l}
\begin{array}{l}
\text { Reserve pool in } \\
\text { bone marrow }
\end{array} \\
\frac{d x_{5}}{d t}=\kappa x_{4}-\psi x_{5} .
\end{array}
\end{aligned}
$$




\section{Lymphopoiesis model}

$$
\begin{array}{ll}
\frac{d x_{1}}{d t}=B x_{1}-x_{1}-\frac{N}{D_{c}} x_{1}, & \frac{d x_{d 2}}{d t}=\frac{N}{D_{2}} \frac{1}{1+\rho_{2}} x_{2}-v_{1} x_{d 2}, \\
\frac{d x_{w d 1}}{d t}=\left(\frac{N}{D_{c}}-\frac{N}{D_{1}}\right) x_{1}+B x_{w d 1}-\psi_{w d 1}, & \frac{d x_{h d 2}}{d t}=\frac{N}{D_{2}} \frac{\rho_{2}}{1+\rho_{2}} x_{1}-v_{2} x_{h d 2} \\
\frac{d x_{d 1}}{d t}=\frac{N}{D_{1}} \frac{1}{1+\rho_{1}} x_{1}-v_{1} x_{d 1}, & \frac{d x_{3}}{d t}=\delta x_{2}-\psi x_{3}-\frac{N}{D_{3}} x_{3}, \\
\frac{d x_{h d 1}}{d t}=\frac{N}{D_{1}} \frac{\rho_{1}}{1+\rho_{1}} x_{1}-v_{2} x_{h d 1}, & \frac{d x_{d 3}}{d t}=\frac{N}{D_{3}} \frac{1}{1+\rho_{3}} x_{3}-v_{1} x_{d 3}, \\
\frac{d x_{2}}{d t}=x_{1}-\delta x_{2}-\frac{N}{D_{2}} x_{2}, & \frac{d x_{h d 3}}{d t}=\frac{N}{D_{3}} \frac{\rho_{3}}{1+\rho_{3}} x_{3}-v_{2} x_{h d 3}
\end{array}
$$




\section{Acute irradiation of human: data of $\mathbf{1 1}$ Chernobyl patients and model simulation}

Exposure range from

2.6 to $7.1 \mathrm{~Sv}$.

Assuming an average 5.9 Sv exposure.

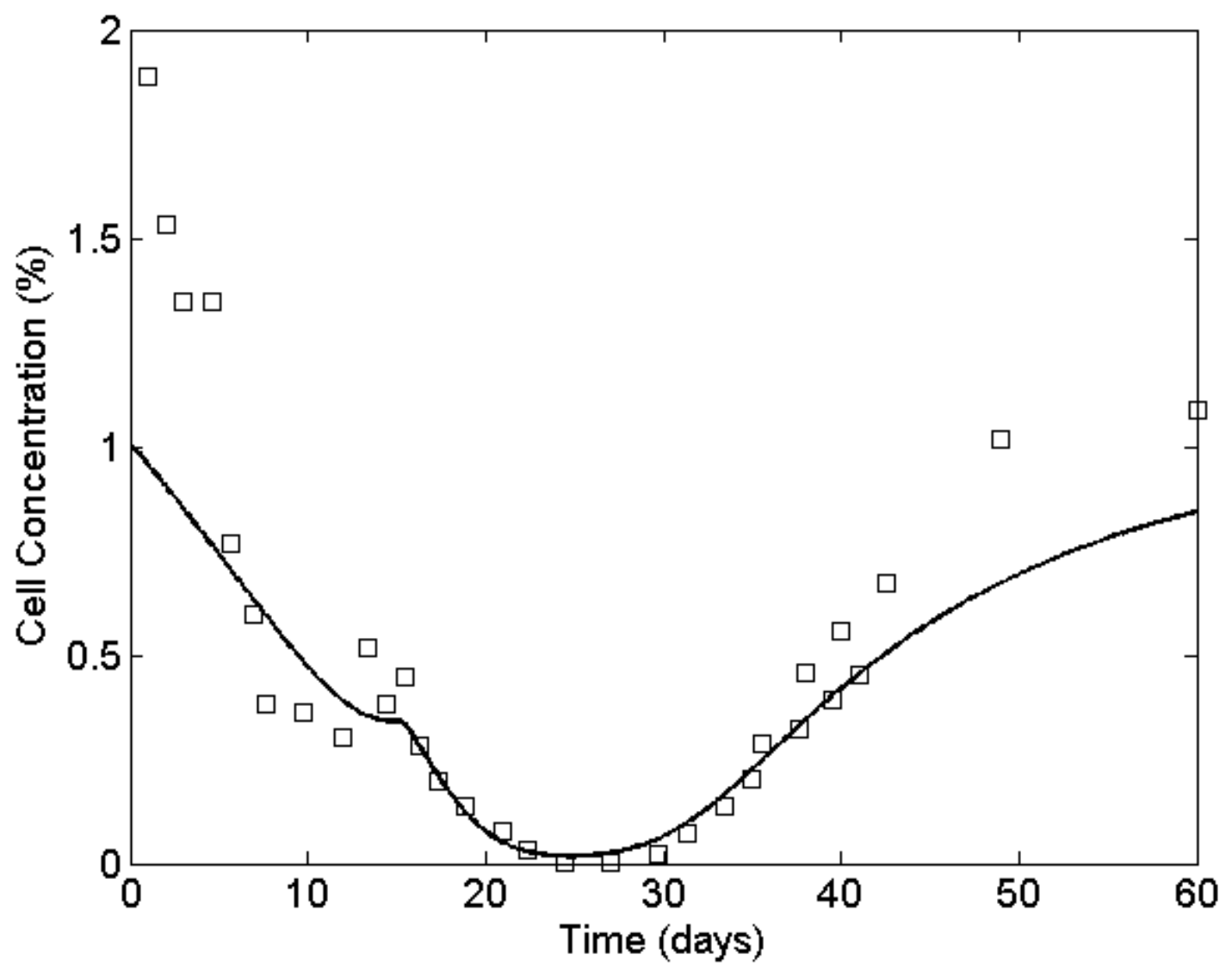




\section{Chernobyl accident}

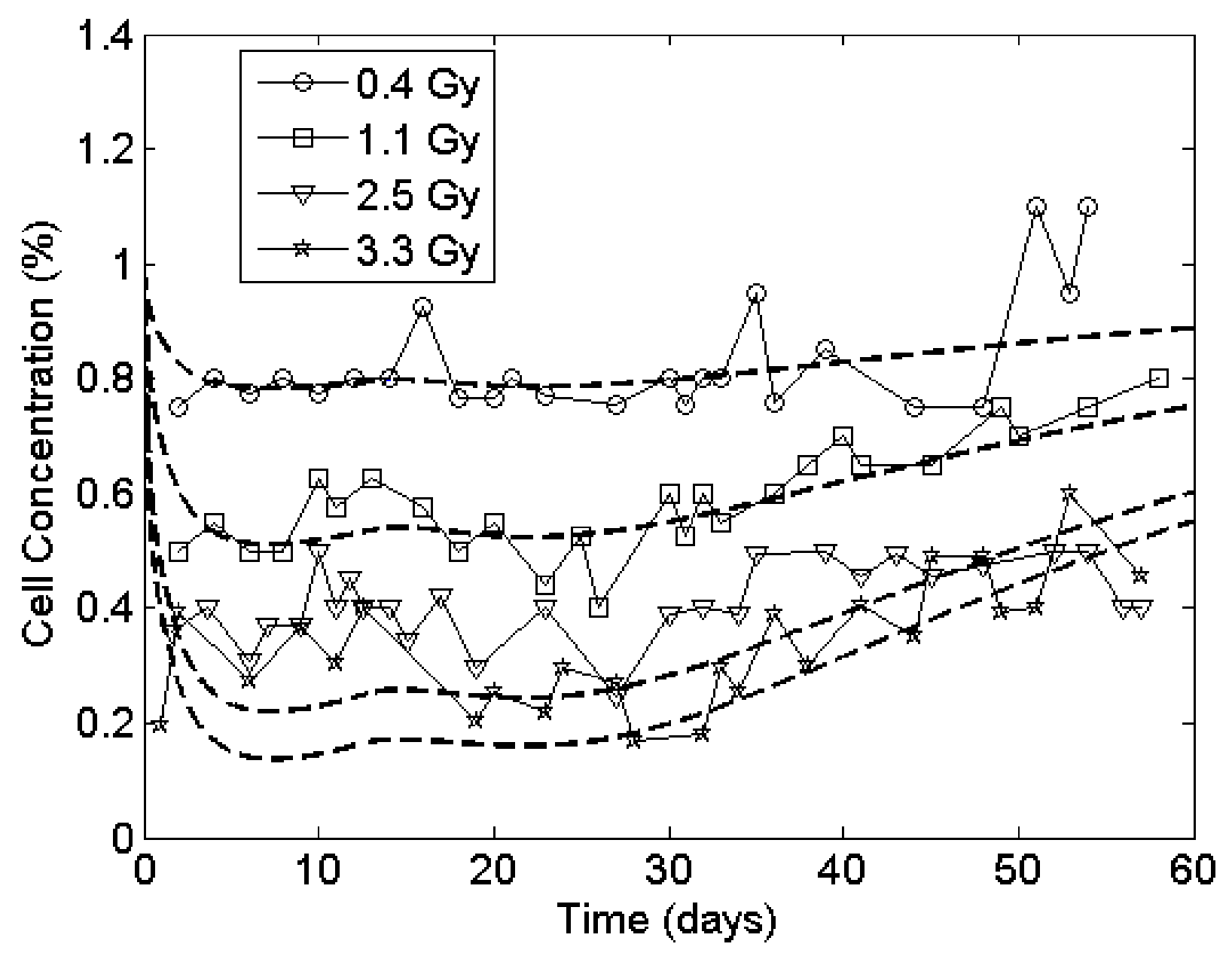

Guskova et al, 2001 


\section{Radiation Exposure from Large SPE Events}

BFO dose rate during Aug. 1972 SPE Event

Cumulative dose

b)
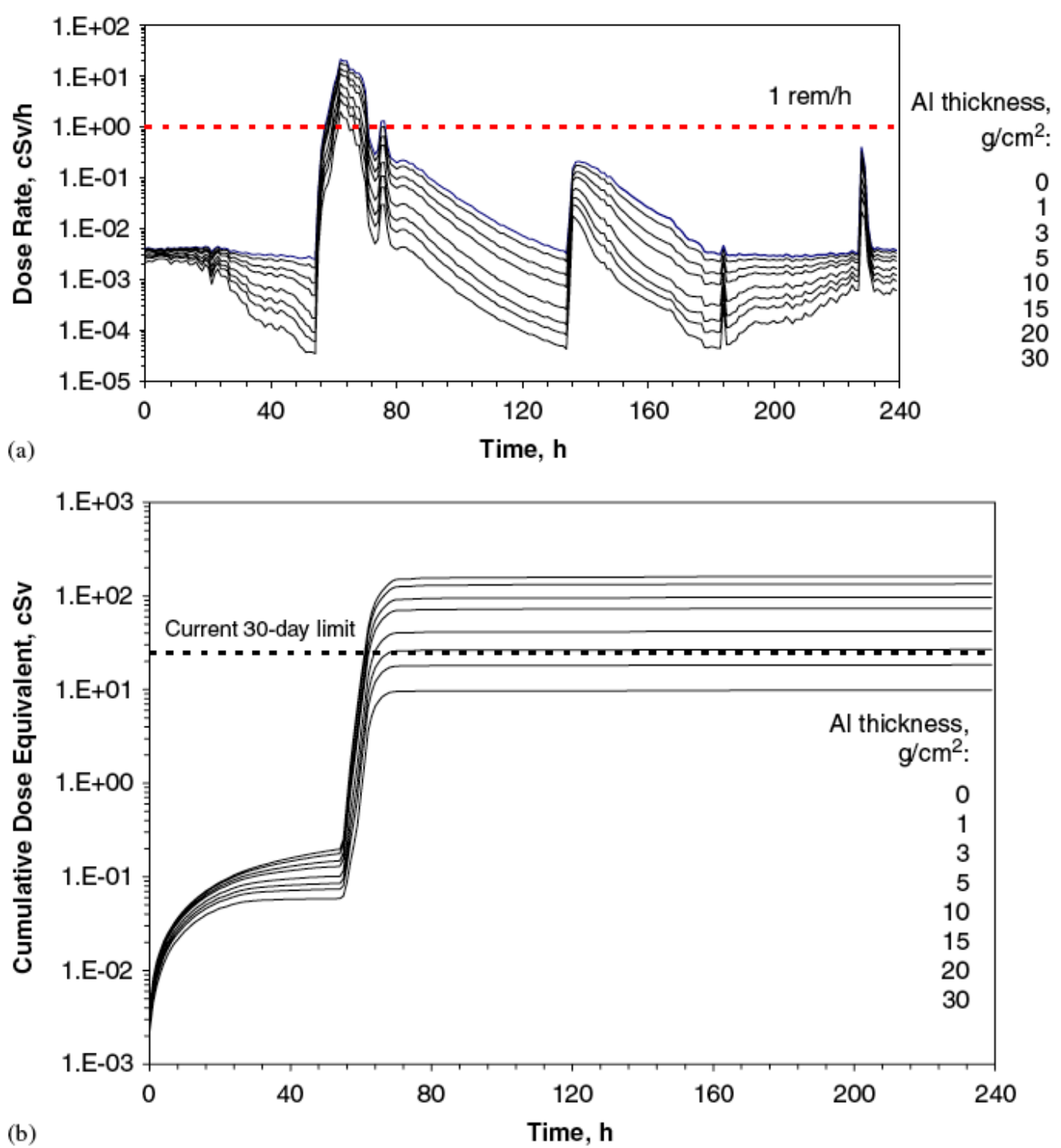

Kim et al., 2006 


\section{Modeling the granulopoiesis response to the 1972 SPE}

Recorded worst SPE: $44.3 \mathrm{mSv} / \mathrm{h}$ for 10 hours inside a typical spacecraft (5 $\mathrm{g} / \mathrm{cm}$ thickness) .

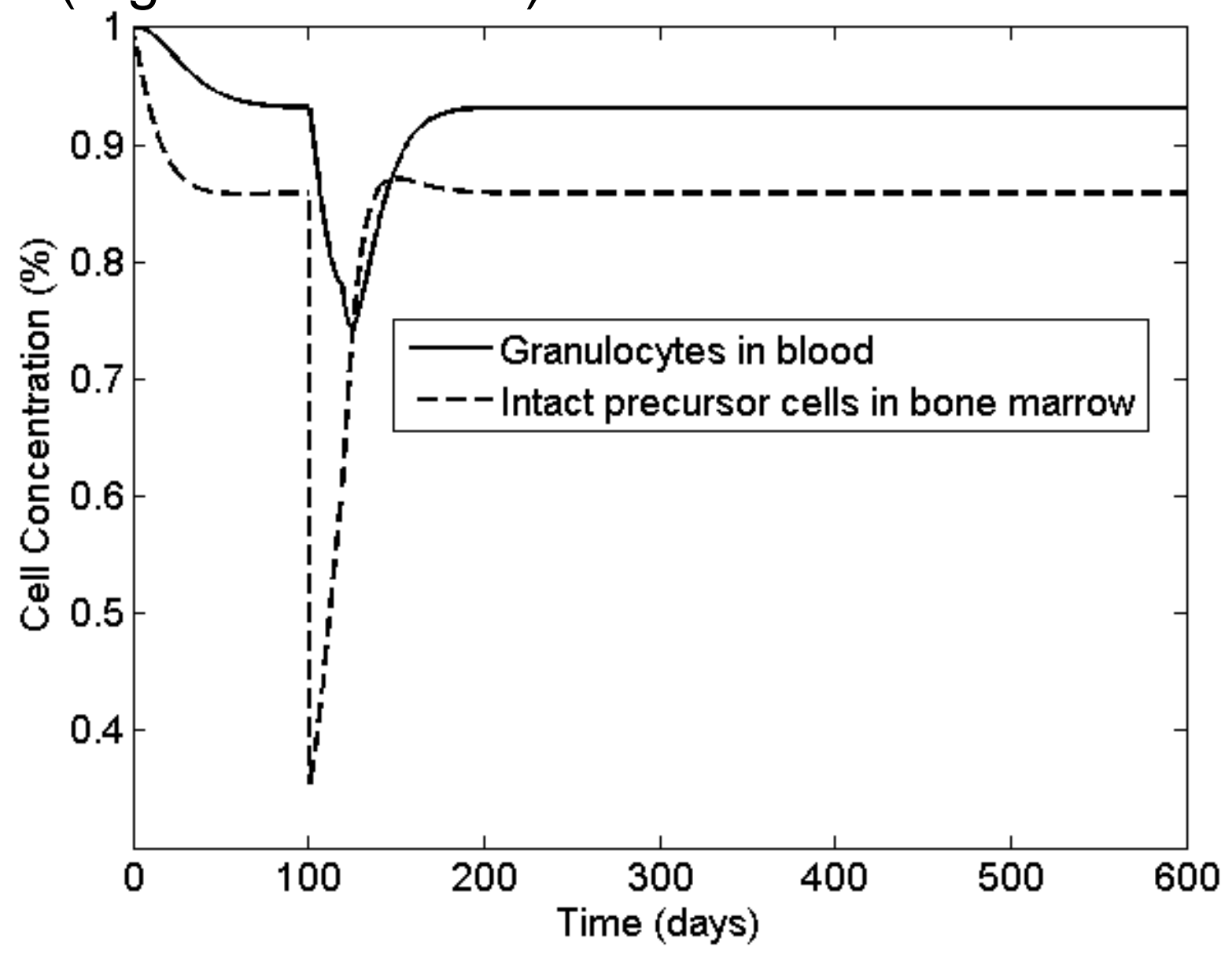




\section{Modeling the lymphopoiesis response to the 1972 SPE}

Recorded worst SPE: $44.3 \mathrm{mSv} / \mathrm{h}$ for 10 hours inside a typical spacecraft (5 g/cm thickness) .

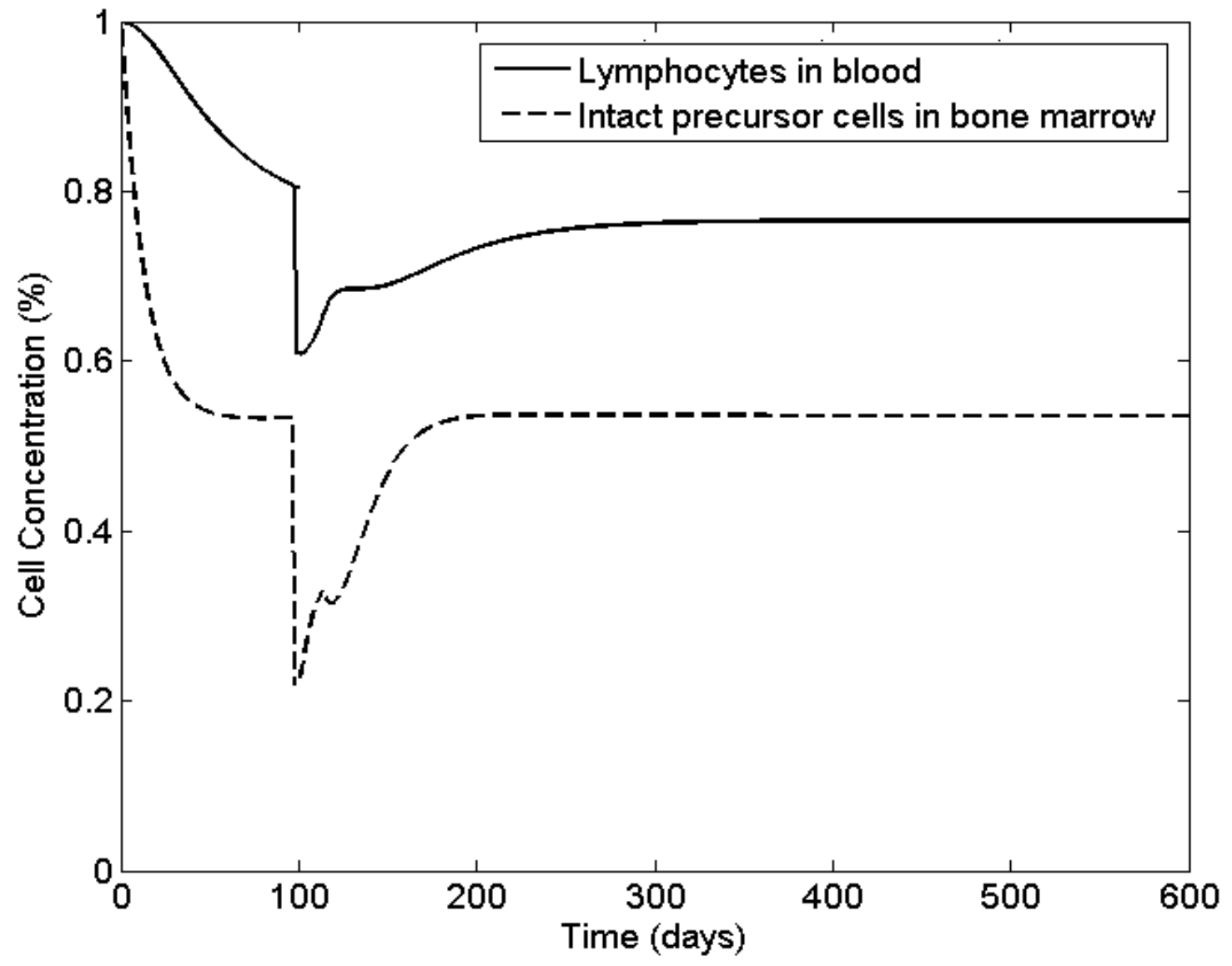




\section{ARRBOD Web Server}

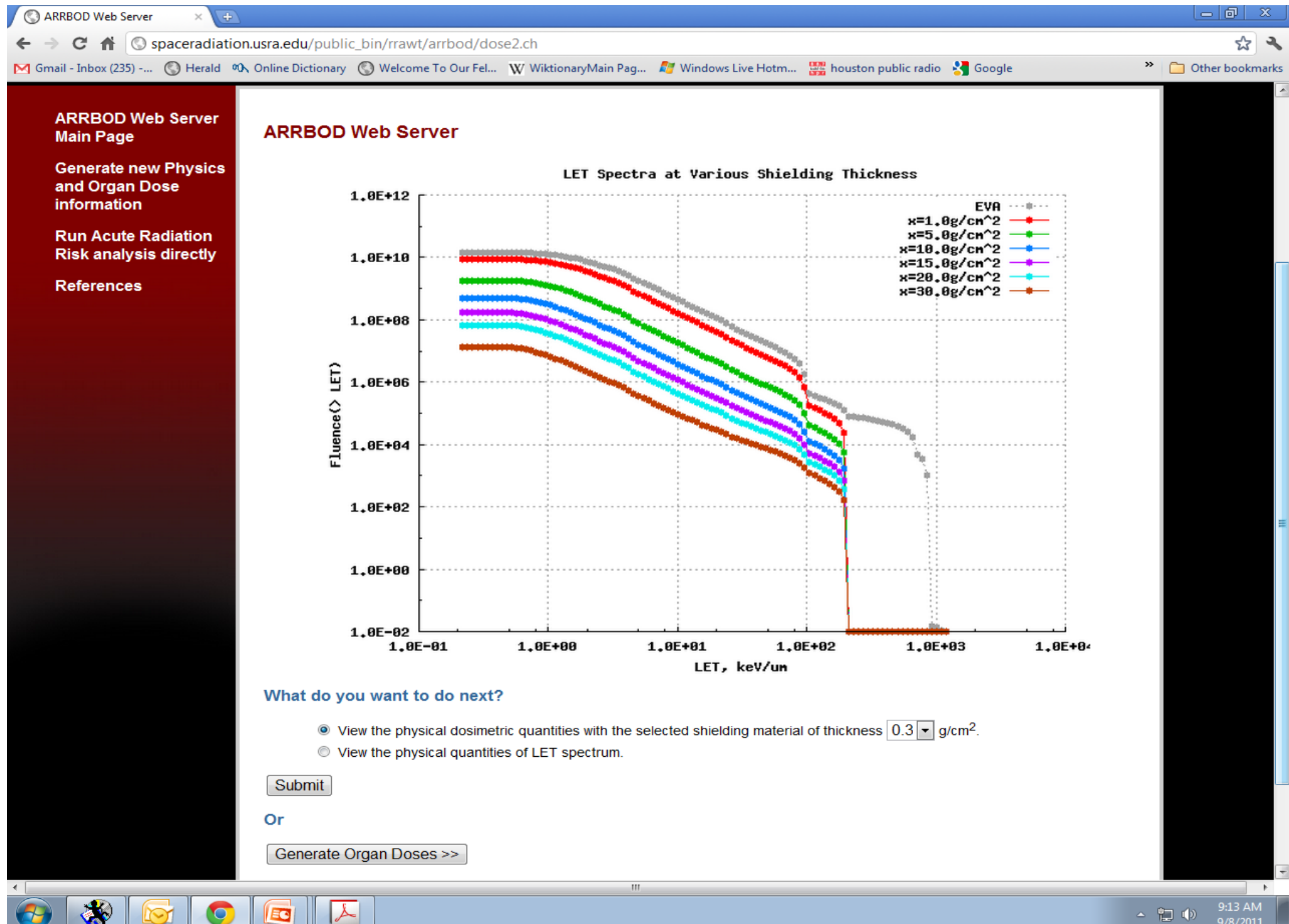

(4)




\section{http://spaceradiation.usra.edu/irModels It is not ready for public use yet.}

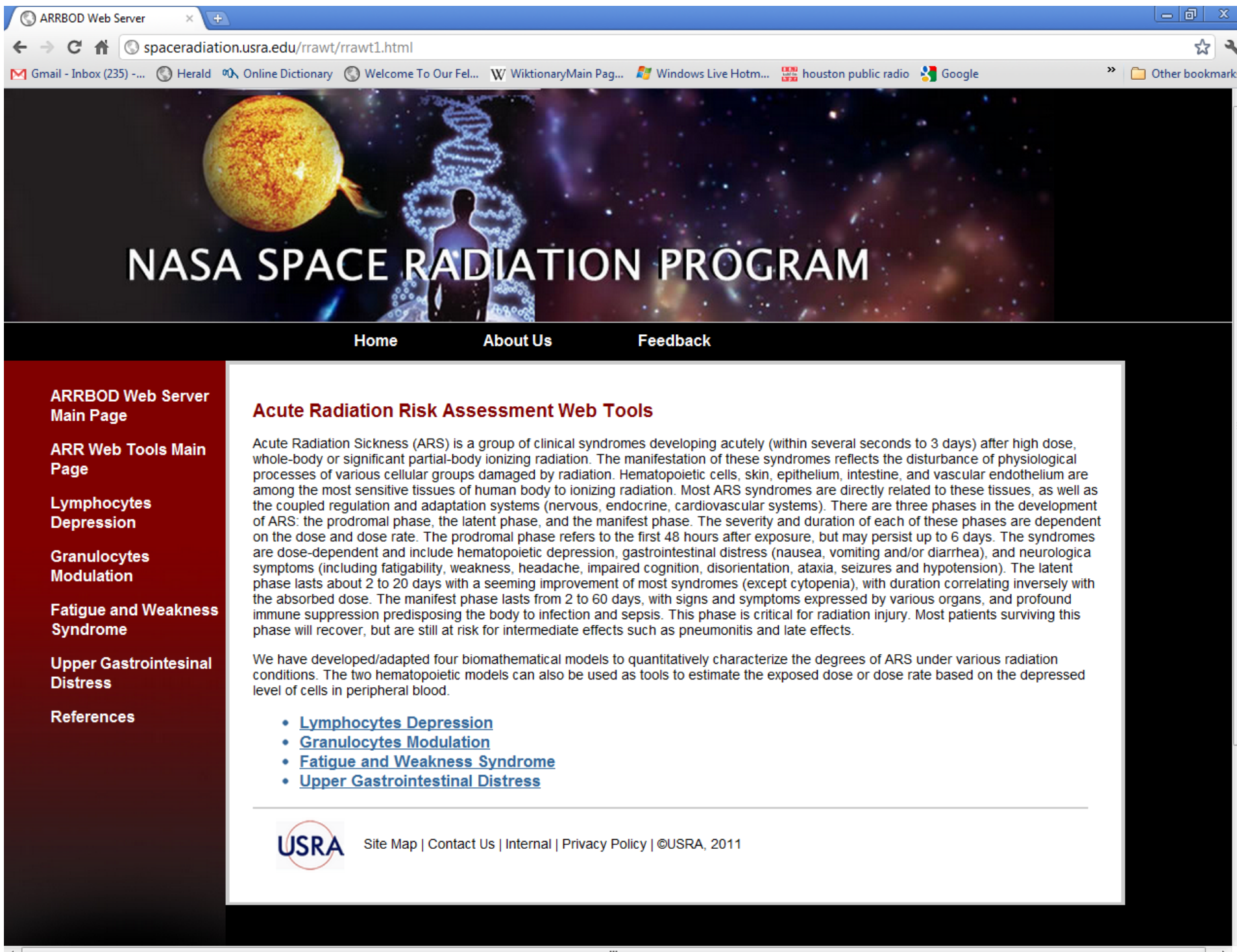




\section{Acknowledgements}

- Dr. Olga Smirnova 